\title{
BEAM-SIZE MEASUREMENTS ON PEP-II USING SYNCHROTRON-LIGHT INTERFEROMETRY
}

\author{
A.S. Fisher, R.L. Holtzapple, M. Petree, and E.L. Bong \\ SLAC, Stanford, CA 94309, U.S.A.
}

\begin{abstract}
PEP-II transverse profiles are measured by imaging visible synchrotron light from dipoles in the two rings. The images are broadened by surface errors on the primary extraction mirrors, which have a complex design due to high beam currents. To improve vertical beam-size measurements, we installed a synchrotron-light interferometer, following Mitsuhashi at KEK. In a two-slit interferometer, fine fringes modulate the single-slit pattern from a point source. As the source size increases, the fringe depth decreases, providing a sensitive tool. Because the slits pass light from two stripes along the mirror, we can select the better parts of its surface. By adding a cylindrical lens to image the mirror in the direction perpendicular to the fringes, we can further select short segments of these stripes. In 2000, we put an interferometer on the low-energy ring, at a point $30 \mathrm{~m}$ from the BaBar detector, where the beam ellipse is tilted as we compensate for rotation in BaBar's solenoid. Our interferometer turns to measure the beam tilt. More recently we added a second unit for the high-energy ring. All optics are in the PEP tunnel and allow remote adjustment of the focusing, slit width and separation.
\end{abstract}

\section{SYNCHROTRON-LIGHT INTERFEROMETRY}

When monochromatic light from a point source diffracts through two parallel slits, the broad fringe pattern from each slit is modulated by fine-scale fringes. Next consider incoherent emission at wavelength $\lambda_{0}$ from a large source $S$. The emitting points in $S$ have uncorrelated phases, and so the fringes from different points overlap and disappear. Between this case and the point source is a transition region known as partial coherence, in which the modulation depth increases from 0 to $100 \%$. The modulation can then be used to measure the source size [1]. A century ago, Michelson used this technique to measure the angular diameter of a star. In recent years, Toshiyuki Mitsuhashi applied it to measuring beam sizes in storage rings at KEK [2].

To find the extent of this transition, consider the light at points $P_{1}$ and $P_{2}$ on the two slits. If for all typical points $S$ in the source, the difference in path length is small compared to a wavelength,

$$
\left|S P_{1}-S P_{2}\right|<<\lambda_{0}
$$

then $P_{1}$ and $P_{2}$ remain correlated. This remains true for a source that is not strictly monochromatic, provided that this path difference is small compared to the coherence length for the bandwidth $\Delta v$ :

$$
c / \Delta v=\lambda_{0}^{2} / \Delta \lambda
$$

We satisfy this condition by inserting a narrow-band optical filter (in our case, a Gaussian centered at $\lambda_{0}=450 \mathrm{~nm}$, with a full width at half maximum of 30 $\mathrm{nm})$. We also add a polarizer in front of the camera, to measure only the horizontal component from the dipole, since the vertical has a sign change at the midplane and therefore shifts the phase of its fringe pattern.

Consider a Gaussian electron or positron beam with a small rms vertical size $\sigma_{y}$. At a distance $s_{0}$ from the source point is a pair of slits of width $a$ and center-tocenter spacing $d$. The slit width is in the $y$ direction and the length is in the $x$ direction. They are symmetrically positioned at $y= \pm d / 2$. The two single-slit diffraction patterns leaving each slit, as a function of the angle $\theta$ to the propagation axis $z$, are:

$$
I_{ \pm}(\theta)=A_{ \pm}\left(\frac{\sin \left[\frac{k a}{2}\left(\theta \mp \frac{d}{2 s_{0}}\right)\right]}{\frac{k a}{2}\left(\theta \mp \frac{d}{2 s_{0}}\right)}\right)^{2} .
$$

A short distance $\Delta s$ beyond the slits, a lens of focal length $f$ directs the light to a screen (actually the CCD chip of a video camera), at a distance $f+\Delta z$ beyond the lens. The optimal distance is the image of the source point, where the two single-slit patterns overlap: The lens maps the angles $\theta$ into heights $y$ on the screen.

We now combine the light from the two slits and apply the theorem of van Cittert and Zernicke [1], which shows that the expression for the fringe pattern is similar to a diffraction integral over the slits, but using the intensity rather than the electric field. We also integrate over the bandpass filter's normalized transmission $g(\lambda)$. The resulting expression

$$
I(y)=
$$

$\int_{-\infty}^{\infty}\left[I_{+}+I_{-}+2 \sqrt{I_{+} I_{-}} \exp \left(-\frac{\left(k d \sigma_{y}\right)^{2}}{2 s_{0}^{2}}\right) \cos \left(\frac{k d y}{f+\Delta z}\right)\right] g(\lambda) d \lambda$

has a cosine factor in the third term, producing the interference fringes, multiplied by an exponential, causing their gradual disappearance with growth in beam size: When the intensity at the two slits is equal, and when the 
camera is on the image plane given by (4), then $I_{+}=I_{-}$, and so (4) can be simplified. Fig. 1 shows typical fringes.

\section{THE PEP-II INTERFEROMETER}

\subsection{PEP-II}

The PEP-II $B$ Factory, a $2.2-\mathrm{km}$ asymmetric collider at the Stanford Linear Accelerator Center [3], was built in collaboration with the Lawrence Berkeley [4] and Lawrence Livermore [5] National Laboratories to study $C P$ violation by tracking decays of $B$ mesons moving in the lab frame. At a single interaction point (IP), $9-\mathrm{GeV}$ electrons in the high-energy ring (HER) collide at zero crossing angle with $3.1-\mathrm{GeV}$ positrons in the low-energy ring (LER). Collisions were first observed in July 1998, and the $B A B A R$ detector was installed in May 1999. Since then the luminosity has increased steadily, reaching a maximum of $3.3 \times 10^{33} \mathrm{~cm}^{-2} \cdot \mathrm{s}^{-1}$, which exceeds the design goal of 3.0. We typically collide $850 \mathrm{~mA}$ of electrons with $1550 \mathrm{~mA}$ of positrons, for a luminosity of 2.8 [6]. Since high luminosity requires a small beam size, its measurement with synchrotron light is an important diagnostic.

\subsection{Limitations of PEP's synchrotron-light mirrors}

The synchrotron-light monitors (SLMs) for the two PEP rings have been described previously [7]. In each, visible light reflected from two in-vacuum mirrors is imaged onto a CCD video camera. The large beam current and consequent high heat load, plus the requirement for a lowimpedance vacuum chamber, led to a primary mirror M1 that is almost flush with the outer wall of the vacuum chamber and hit at grazing incidence. A 4-mm-high slot along the mirror's midplane passes the hot x-ray fan, while visible light reflects from the surfaces above and below. Due to grazing incidence, the $\mathrm{x}$ rays never reach the bottom of the slot, but travel past the mirror to dump their heat into a thermally separate absorber. Extensive water-cooling channels in M1 absorb the heat and prevent damage when the fan is not aligned with the slot.

The M1 mirrors for HER and LER were initially flat when polished, but once installed their images did not approach the diffraction limit. Subsequent investigation indicated that problems arose after polishing, when the long cooling tubes were welded to short tubing stumps on

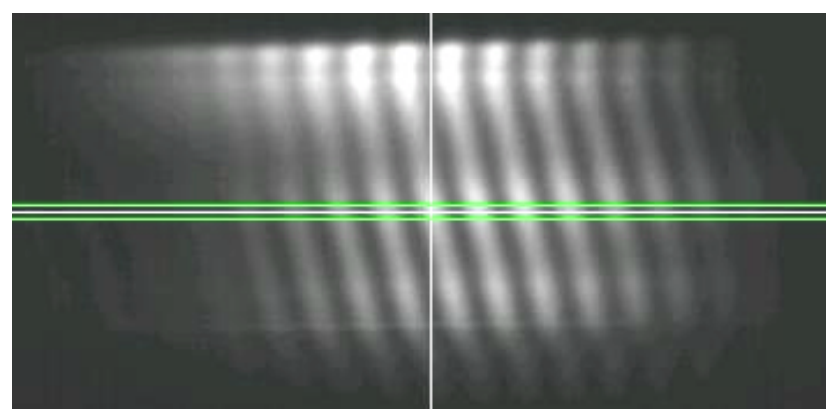

Figure 1: Fringes from the HER. The rectangle indicates the region selected for the curve fit. the rear surface. Further stresses appear to arise from the stiffness and weight of the tubes, and perhaps also from the mount holding the mirror in the vacuum chamber.

\subsection{Imaging for M1 Compensation}

Our interferometer adds compensation for the nonuniformity of M1. The two slits transmit light from two narrow stripes along the mirror. When measuring $\sigma_{y}$, the slits and stripes are parallel to $x$, thus selecting two $y$ coordinates on the mirror. The fringe pattern then consists of horizontal lines imposed on an image of the beam.

We can be more selective by imaging M1 - rather than the source point-along $x$. A cylindrical lens, placed downstream of the primary (spherical) lens, shortens the focal length in $x$ to image M1 onto the camera. Thus on the video screen, the $x$ coordinates correspond to different $x$ positions on the mirror, but the $y$ coordinates still show the interference fringes from the source point. By digitizing the image and selecting on the computer the range of $x$ with the clearest fringes, we have limited our use of M1 to two small rectangles.

The typical fringes from the HER in Fig. 1 are not quite as described above. A periscope has rotated the pattern by $90^{\circ}$, and the fringes are noticeably tilted. The tilt indicates a phase change along the mirror's surface: in following the two stripes along $x$, the optical-path difference at the slits varies by about one wavelength. This effect appears in the interferogram of Fig. 2, taken when the mirror was inspected after the first year of operation. The interferometer also provides on-line mirror-quality analysis. Without a cylindrical lens imaging M1 and allowing selective analysis, the fringe pattern would mostly disappear.

\subsection{Controls, Acquisition, and Analysis}

Because each interferometer is located in the tunnel, it requires several motors for remote control. A pair of motorized mirrors steers the light through the slits and to the camera. The camera and cylindrical lens translate for focusing. At the LER source point, $30 \mathrm{~m}$ from $B A B A R$, the beam ellipse is tilted as we compensate for rotation in $B A B A R$ 's solenoid. The interferometer turns to measure the beam tilt, and the cylindrical lens turns with it so that its focusing is in the correct plane.

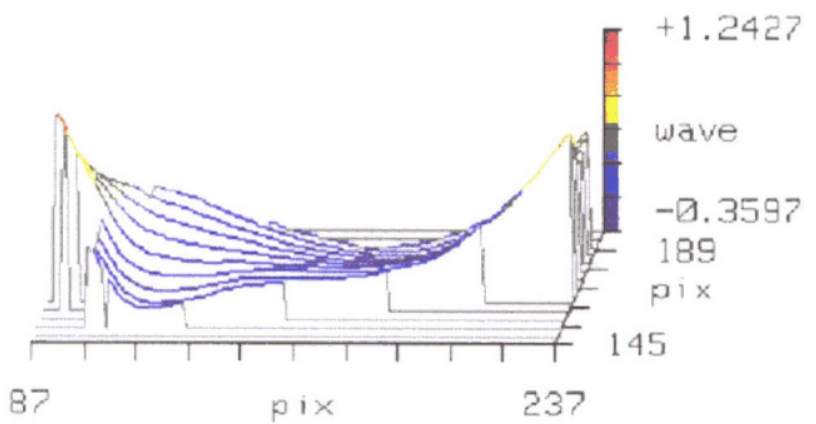

Figure 2: A profile one half of the surface of HER's M1, in units of HeNe-laser wavelengths, showing the skew of the surface normal. 


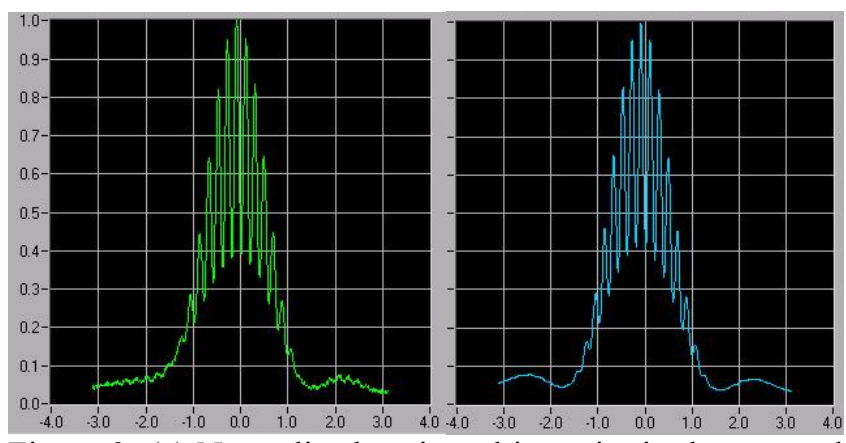

Figure 3: (a) Normalized projected intensity in the rectangle of Fig. 1, vs. position in $\mathrm{mm}$ on the CCD. (b) Calculated curve fit, giving an rms size of $232 \mu \mathrm{m}$.

A PC with a video frame-grabber card acquires the image of the fringes. A LabVIEW program then allows the user to select a rectangular region for analysis (Fig. 1). The intensity pattern is projected onto the horizontal axis, and the program performs a least-squares nonlinear curve fit to match the projection of the fringe pattern to the theoretical curve and so determine the beam size. Fig. 3(a) plots the projected intensity of the data in the rectangle of Fig. 1, and Fig. 3(b) shows the calculated fit.

\section{BEAM SIZE MEASURMENTS}

We scanned the HER's transverse tunes across the coupling resonance $\left(v_{x}=v_{y}\right)$ while measuring the vertical beam size with both the interferometer and SLM. The experiment was made at low current with colliding beams. Every $10 \mathrm{sec}$, we measured the two independent beam sizes, the specific luminosity and the beam lifetime. The tunes, read manually from a spectrum analyzer, were slowly varied from $(0.58,0.65)$, starting with the horizontal followed by the vertical. When the horizontal tune approached 0.60 , the lifetime and specific luminosity dropped, indicating a resonance (Fig. 4). Both recovered afterward, but as the tunes approached the coupling

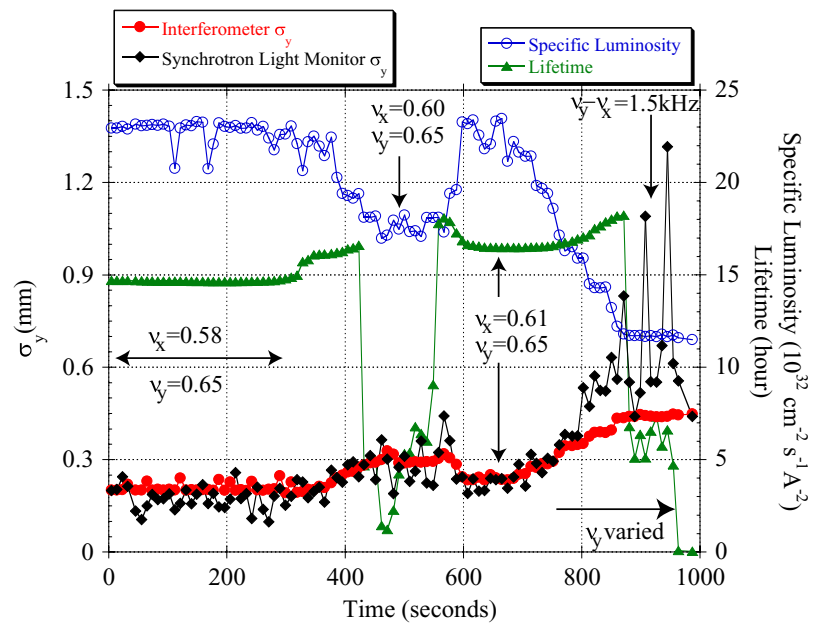

Figure 4: The specific luminosity, the HER lifetime, and the HER interferometer and SLM beam sizes, measured every $5 \mathrm{~s}$ while the tunes were varied. The SLM data has been corrected for the resolution of the camera. resonance, the specific luminosity quickly dropped, and the beams were lost when the tune separation reached approximately $0.011(1.5 \mathrm{kHz})$.

Fig. 4 correlates the vertical beam size measured by the interferometer and the SLM with the specific luminosity. The comparison provided a measurement of the resolution of the synchrotron-light monitor: subtracting $0.345 \mathrm{~mm}$ in quadrature from the SLM data gives good agreement with the interferometer. Between the first resonance the coupling resonance, both sizes increased by more than a factor of two. Also, Fig. 5 shows the expected reduction in beam size as the specific luminosity increases.

Thus the interferometer is proving to be an excellent beam-size diagnostic. Compared to the SLM, it can resolve smaller sizes with less impact from distortion in the primary mirror. The measurements will prove useful as we attempt to further increase PEP's luminosity.

\section{REFERENCES}

[1] Born, M., and Wolf, E., Principles of Optics, $5^{\text {th }}$ ed. (Pergamon Press, Oxford, 1975).

[2] Mitsuhashi, T., "Beam Profile and Size Measurement by SR Interferometers," in Beam Measurement, Proc. Joint US-CERN-Japan-Russia School on Particle Accelerators, Montreux, Switzerland, 11-20 May 1998 (World Scientific, Singapore), pp. 399-427.

[3] U.S. Dept. of Energy contract DE-AC03-76SF00515.

[4] U.S. Dept. of Energy contract DE-AC03-76SF00098.

[5] U.S. Dept. of Energy contract W-7405-Eng-48.

[6] Seeman, J., in these Proceedings.

[7] Fisher, A.S., "Instrumentation and Diagnostics for PEP-II," Proc. Beam Instrumentation Workshop, Stanford, CA, 4-7 May 1998, AIP Conf. Proc. 451 (Amer. Inst. Phys., Woodbury, NY, 1998), pp. 395-403.

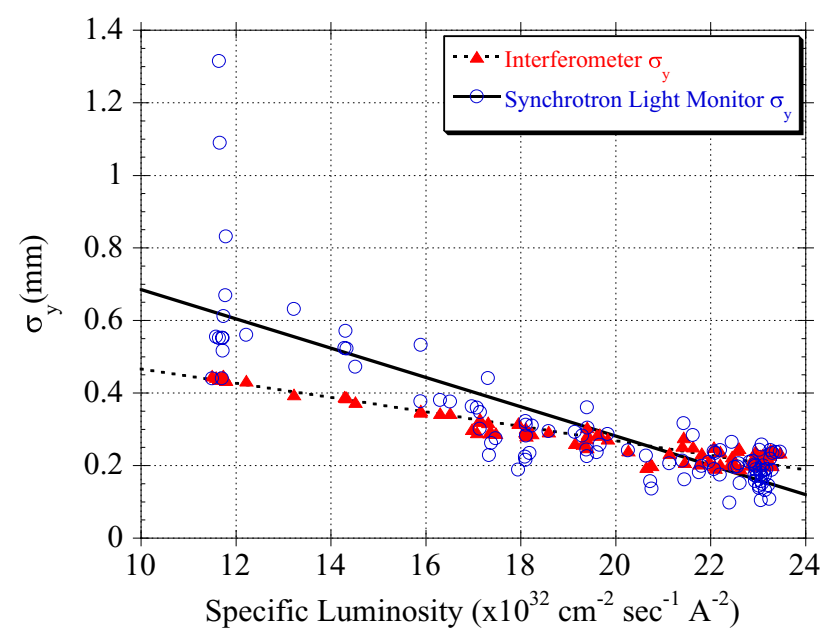

Figure 5: HER vertical beam size, measured by both the interferometer and SLM, vs. specific luminosity. 